\title{
Microflow Simulations via the Lattice Boltzmann Method
}

\author{
Nikolaos Prasianakis ${ }^{1, *}$ and Santosh Ansumali ${ }^{2}$ \\ ${ }^{1}$ Combustion Research, Paul Scherrer Institute, Villigen PSI 5232, Switzerland. \\ ${ }^{2}$ Engineering Mechanics Unit, Jawaharlal Nehru Centre for Scientific Research, \\ JNCASR, 560064 Bangalore, India.
}

Received 30 October 2009; Accepted (in revised version) 27 October 2010

Available online 14 January 2011

\begin{abstract}
The exact solution to the hierarchy of nonlinear lattice Boltzmann kinetic equations, for the stationary planar Couette flow for any Knudsen number was presented by S. Ansumali et al. [Phys. Rev. Lett., 98 (2007), 124502]. In this paper, simulation results at a non-vanishing value of the Knudsen number are compared with the closed-form solutions for the higher-order moments. The order of convergence to the exact solution is also studied. The lattice Boltzmann simulations are in excellent agreement with the exact solution.
\end{abstract}

AMS subject classifications: 52B10, 65D18, 68U05, 68U07

Key words: Microflows, lattice Boltzmann method.

\section{Introduction}

Engineering interest in microelectromechanical systems (MEMS) has experienced explosive growth during the last two decades [1]. These kind of devices typically have characteristic size ranging from a few micron to a few millimeters, often with high aspect ratios. Often one is interested in gaseous flows through micro-devices such as micro-pumps, micro-turbines, and micro-valves [1]. It is well understood that for gaseous flows at those length-scales hydrodynamic framework starts to breakdown. A computational approach that recently emerged as a potential candidate for such gaseous micro-flow simulations is the lattice Boltzmann method (LB).

This computational tool has been also used to study many other microfluidic flow problems involving multiphase flows [3-7]. In all these studies, the role of proper wall boundary conditions has been shown to be essential. In this paper, the ability of the method to capture numerically, non-trivial micro-flow phenomena for single component gaseous flows, by using the diffusive boundary condition [8] is studied in detail.

\footnotetext{
${ }^{*}$ Corresponding author. Email addresses: nikolaos.prasianakis@psi.ch (N. I. Prasianakis), ansumali@
} gmail. com (S. Ansumali) 


\section{Lattice Boltzmann approach for microflows}

The capability of the lattice Boltzmann method to simulate micro-flows stems from its kinetic theory origin. The lattice Boltzmann method is a special velocity-time-space discretization of the continuous Boltzmann BGK equation.

Even though the solution of the discrete Boltzmann BGK equation in the bulk flow is quite standard,for the implementation of the wall boundary condition several approaches exist $[2,5,8]$. The most commonly used ones are the bounce back and the kinetic diffusive boundary condition. In this paper we focus on the study of the numerical discretization of the diffusive boundary condition as presented in $[8,9]$. One of the most attractive features of this boundary condition is the non-trivial micro-flow effects that can be captured without the need of tuning any parameters. For simplicity the nine-velocities, two-dimension lattice BGK model (D2Q9) will be considered. The microCouette flow is the selected micro-flow setup case. Analytical solutions for that problem is reported in [10,11]. For that, the moment system of the Boltzmann BGK equation [12], combined with the properties of the D2Q9 square lattice discretization are analyzed. We consider the popular nine-velocity model, the so-called D2Q9 lattice, for which the discrete velocities are: $c_{0}=(0,0), c_{i}=( \pm 1,0)$ and $(0, \pm 1)$, for $i=1-4$, and $c_{i}=( \pm 1, \pm 1)$, for $i=5-8$ (see [14]). The discrete lattice Bhatnagar-Gross-Krook (BGK) equation reads:

$$
\partial_{t} f_{i}+c_{i \alpha} \partial_{\alpha} f_{i}=-\frac{1}{\tau}\left(f_{i}-f_{i}^{e q}\right),
$$

where $\tau$ is the relaxation parameter, and $f_{i}^{e q}$ the values of populations at equilibrium. The equilibrium distribution functions neglecting terms of the order $\mathcal{O}\left(u^{3}\right)$ and higher can be expressed as:

$$
f_{i}^{e q}=w_{i}\left(\rho+\frac{j_{\alpha} c_{i \alpha}}{c_{S}^{2}}+\frac{j_{\alpha} j_{\beta}}{2 \rho c_{S}^{4}}\left(c_{i \alpha} c_{i \beta}-c_{S}^{2} \delta_{\alpha \beta}\right)\right),
$$

where $c_{s}$ is the speed of sound, $c_{s}=\sqrt{\left(k_{B} T_{0}\right) / m}=\sqrt{1 / 3}$. For the D2Q9 model, the corresponding weights $w_{i}$ are:

$$
w=\frac{1}{36}\left[\begin{array}{lllllllll}
16 & 4 & 4 & 4 & 4 & 1 & 1 & 1 & 1
\end{array}\right] .
$$

In the hydrodynamic limit, this model recovers the Navier-Stokes equations with the kinematic viscosity $v=\tau c_{s}^{2}$. By considering the moments of the lattice-BGK equation, a moment system can be created and closed form solutions for specific flow setups can be obtained.

\section{Moment system}

Since the D2Q9 lattice is described by a set of nine distribution functions $f_{i}$, a ninemoment system will be formulated. For the isothermal D2Q9 model, the conserved fields 
during the collision process are mass density $\rho$ and momentum density $j_{\alpha}$ defined as

$$
\rho=\sum f_{i}, \quad j_{x}=\sum c_{i x} f_{i}, \quad j_{y}=\sum c_{i y} f_{i} .
$$

It is convenient to re-write the pressure tensor $P_{\alpha \beta}=\sum c_{i \alpha} c_{i \beta} f_{i}$ in terms of the trace $P$, the normal stress difference $N$, and off-diagonal term of the pressure tensor $P_{x y}$ defined as

$$
P \equiv P_{x x}+P_{y y}=\sum c_{i}^{2} f_{i}, \quad N \equiv P_{x x}-P_{y y}=\sum f_{i}\left(c_{i x}^{2}-c_{i y}^{2}\right), \quad P_{x y}=\sum c_{i x} c_{i y} f_{i} .
$$

Furthermore, the flux of energy is defined as

$$
q_{\alpha}=\sum c_{i \alpha} c_{i}^{2} f_{i}
$$

In order to close the system in terms of moments, one more independent moment $\psi$ is defined as

$$
\psi=\sum f_{i} c_{i x}^{2} c_{i y}^{2}
$$

The time evolution of the conserved hydrodynamic fields using Eq. (2.1) are

$$
\begin{aligned}
& \partial_{t} \rho+\partial_{x} j_{x}+\partial_{y} j_{y}=0, \\
& \partial_{t} j_{x}+\partial_{x}\left(\frac{P+N}{2}\right)+\partial_{y} P_{x y}=0, \\
& \partial_{t} j_{y}+\partial_{x} P_{x y}+\partial_{y}\left(\frac{P-N}{2}\right)=0 .
\end{aligned}
$$

Similarly, the evolution equations for the pressure tensor are

$$
\begin{aligned}
& \partial_{t} P+\partial_{x} q_{x}+\partial_{y} q_{y}=\frac{1}{\tau}\left(2 \rho c_{s}^{2}+\frac{j^{2}}{\rho}-P\right), \\
& \partial_{t} P_{x y}+\partial_{x}\left(q_{y}-\frac{3 k_{\mathrm{B}} T_{0}}{m} j_{y}\right)+\partial_{y}\left(q_{x}-\frac{3 k_{\mathrm{B}} T_{0}}{m} j_{x}\right)=\frac{1}{\tau}\left(\frac{j_{x} j_{y}}{\rho}-P_{x y}\right), \\
& \partial_{t} N+\partial_{x}\left(\frac{6 k_{\mathrm{B}} T_{0}}{m} j_{x}-q_{x}\right)+\partial_{y}\left(q_{y}-\frac{6 k_{\mathrm{B}} T_{0}}{m} j_{y}\right)=\frac{1}{\tau}\left(\frac{j_{x}^{2}}{\rho}-\frac{j_{y}^{2}}{\rho}-N\right) .
\end{aligned}
$$

The equation of motion for the third order moments are

$$
\begin{aligned}
& \partial_{t} q_{x}+\partial_{x}\left(\psi+\frac{3 k_{\mathrm{B}} T_{0}}{2 m}(P+N)\right)+\frac{6 k_{\mathrm{B}} T_{0}}{m} \partial_{y} P_{x y}=\frac{1}{\tau}\left(4 c_{s}^{2} j_{x}-q_{x}\right), \\
& \partial_{t} q_{y}+\frac{6 k_{\mathrm{B}} T_{0}}{m} \partial_{x} P_{x y}+\partial_{y}\left(\psi+\frac{3 k_{\mathrm{B}} T_{0}}{2 m}(P-N)\right)=\frac{1}{\tau}\left(4 c_{s}^{2} j_{y}-q_{y}\right) .
\end{aligned}
$$

Finally, the evolution equation for $\psi$ is

$$
\partial_{t} \psi+\frac{3 k_{\mathrm{B}} T_{0}}{m} \partial_{x}\left(q_{x}-\frac{3 k_{\mathrm{B}} T_{0}}{m} j_{x}\right)+\frac{3 k_{\mathrm{B}} T_{0}}{m} \partial_{y}\left(q_{x}-\frac{3 k_{\mathrm{B}} T_{0}}{m} j_{y}\right)=\frac{1}{\tau}\left(\rho c_{s}^{4}+\frac{j^{2}}{\rho} c_{s}^{2}-\psi\right) .
$$


The present moment system has non-trivial analytical solutions for simple flows, such as the stationary Couette flow [10]. Before proceeding to the analytic solution of the moment system for the case of the Couette flow, the diffusive boundary condition will be outlined.

The characteristic feature of the diffusive boundary condition is that the particles, after reaching the solid boundary, get redistributed in a way consistent with the massbalance and normal-flux conditions. Furthermore, the boundary condition satisfies the condition of detailed balance. In other words, if the incoming populations are at equilibrium (corresponding to the boundary-velocity), the outgoing populations are also at equilibrium (corresponding to the boundary-velocity). For what it follows we consider a flat wall along the $x$-direction as depicted in Fig. 1.

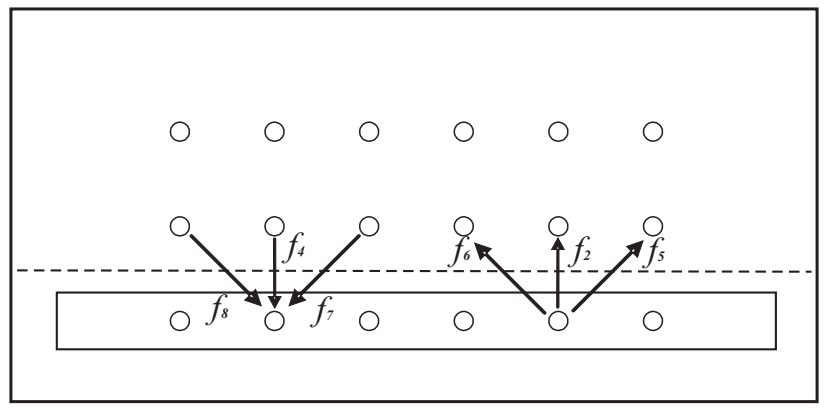

Figure 1: Schematic plot of the interaction of the diffusive boundary wall with the fluid. Solid wall is placed at the lower part of the domain. Populations $f_{4}, f_{7}, f_{8}$ are the incoming populations and $f_{2}, f_{5}, f_{6}$ are the outgoing populations which can be computed with the help of Eq. (3.9).

The update rules for the outgoing populations which are affected by the velocity of the wall $\mathbf{u}_{\text {wall }}$ are:

$$
\begin{aligned}
& f_{2}(\mathbf{x}, t)=f_{2}^{e q}\left(\rho, \mathbf{u}_{\text {wall }}\right) G, \\
& f_{5}(\mathbf{x}, t)=f_{5}^{e q}\left(\rho, \mathbf{u}_{\text {wall }}\right) G, \\
& f_{6}(\mathbf{x}, t)=f_{6}^{e q}\left(\rho, \mathbf{u}_{\text {wall }}\right) G,
\end{aligned}
$$

with $G$ defined as:

$$
G=\frac{f_{4}(\mathbf{x}, t)+f_{7}(\mathbf{x}, t)+f_{8}(\mathbf{x}, t)}{f_{2}^{e q}\left(\rho, \mathbf{u}_{\text {wall }}\right)+f_{5}^{\text {eq }}\left(\rho, \mathbf{u}_{\text {wall }}\right)+f_{6}^{e q}\left(\rho, \mathbf{u}_{\text {wall }}\right)} .
$$

In the next section, the case of the isothermal Couette flow is considered.

\section{Stationary Couette flow}

The planar Couette flow, between two parallel plates separated by a distance $L$ has been studied in [10]. The plates are parallel to the $x$-axis. On the plates, the isothermal diffusive 


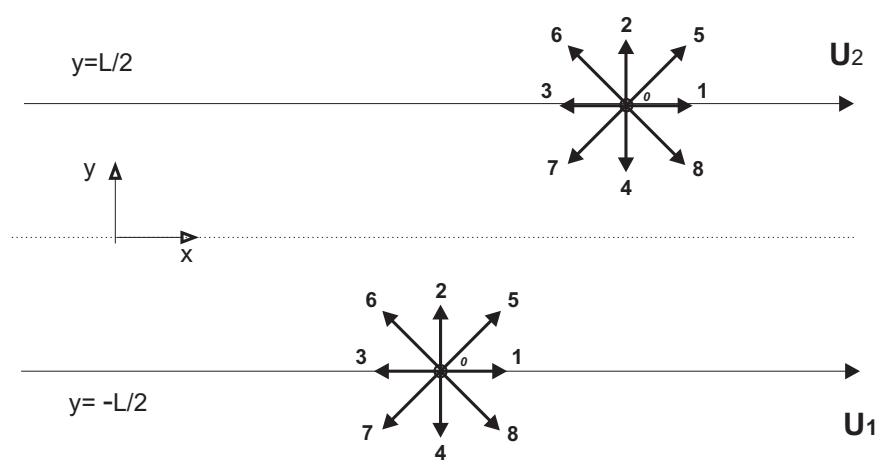

Figure 2: Schematic plot of the Couette flow. Periodicity is assumed in the $x$-axis. The top and bottom plates are moving parallel to the $x$-axis with velocities $U_{2}$ and $U_{1}$ respectively.

boundary condition is implemented. The exact implementation used in this paper is presented in $[8,9]$. Periodic boundary condition is applied on the first and the last column of nodes in the $x$-direction. The flow is unidirectional and all hydrodynamic quantities are $y$-coordinate dependent. Under these assumptions, the moment system of Eqs. (3.5a)(3.5c) is drastically simplified and the expression for the velocity profile along the $x$-axis reads:

$$
u_{x}(y)=\frac{j_{x}}{\rho}=-\frac{\pi^{n e q}}{\tau c_{s}^{2}}(y+\tau V)
$$

The expressions of the constants $\pi^{n e q}=P_{x y}^{n e q} / \rho$ and $V$ can be found using the closure relations provided by the diffusive boundary condition. It is noted that a given moment of the populations, i.e., $M$, can be always expressed as the equilibrium part, plus a nonequilibrium part: $M=M^{e q}+M^{n e q}$. Introducing the mean free path $l$ as $l=\sqrt{3} \tau c_{s}$ and the Knudsen number $K n=l / L$, which is defined as the ratio of the mean free path $l$ over the characteristic size of the studied system, their form has been found to be:

$$
\begin{aligned}
& \pi^{n e q}=-\frac{\tau c_{s}^{2} \Delta U}{4\left(2 \sqrt{3} \tau c_{s}+L\right)}, \\
& V=\frac{\left(2 \sqrt{3} \tau c_{s}+L\right)}{\tau} \frac{U}{\Delta U^{\prime}}
\end{aligned}
$$

where $U=\left(U_{2}+U_{1}\right) / 2$, and $\Delta U=U_{2}-U_{1}$, as explained in Fig. 2. The Eq. (4.1) receives the final form:

$$
u_{x}(y)=\frac{\Delta U}{(2 K n+1)} \frac{y}{L}+U
$$

The above description of the velocity field for the stationary isothermal Couette flow predicts non-trivial slip velocity effects dependent on the Knudsen number Kn. In Fig. 3, the analytic slip velocity on the upper wall (4.3) is represented by the solid line. This 


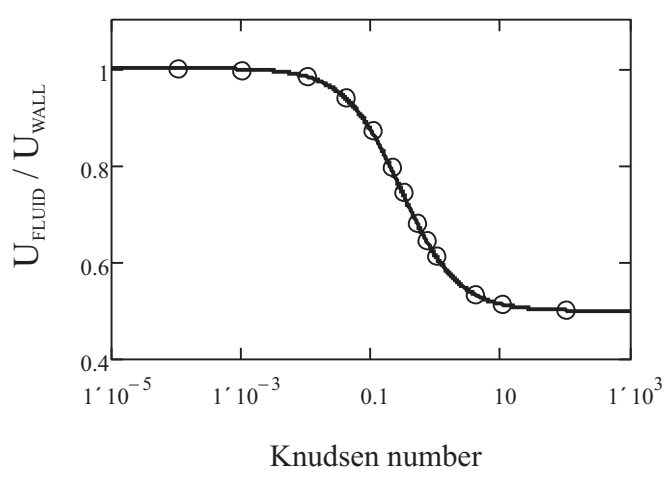

Figure 3: Stationary isothermal Couette flow. Slip velocity for the upper wall. Line: exact solution; Symbol: lattice Boltzmann simulation.

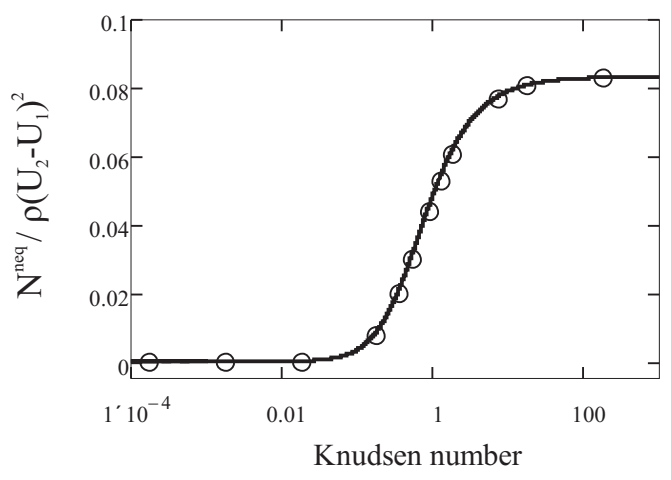

Figure 4: Non-equilibrium normal stress difference $N^{\text {neq }}$ at the upper moving wall. Line: analytic solution; Symbol: lattice Boltzmann simulation.

result is compared with the lattice Boltzmann simulations (symbols) for a large range of Knudsen numbers.

Using Eqs. (3.6a)-(3.8), the expressions for the non-equilibrium stress difference $N^{\text {neq }}$ and for the non-equilibrium heat flux $q_{y}^{\text {neq }}$ can also be expressed in an exact form:

$$
\begin{aligned}
& N^{n e q}(y)=\rho\left(\frac{\Delta U}{L}\right)^{2} \frac{\tau v}{(1+2 K n)^{2}}\left[2-e^{-\frac{1}{2 K n}} \cosh \left(\frac{y}{K n L}\right)\right] \\
& q_{y}^{n e q}(y)=-\rho\left(\frac{\Delta U}{L}\right)^{2} \frac{v}{(1+2 K n)^{2}}\left[2 y-K n L e^{-\frac{1}{2 K n}} \sinh \left(\frac{y}{K n L}\right)\right]+2 U P_{x y}^{n e q} .
\end{aligned}
$$

In Fig. 4, the comparison between Eq. (4.4a) and lattice Boltzmann simulations at the upper wall, $y=L / 2$ is plotted. A non-trivial behavior of the higher order moments is revealed. This behavior is well-captured by the LB simulations.

\section{Simulation results}

In the previous section, the analytical solutions of the micro-Couette flow simulation results concerning the slip velocity, and the behavior of the normal stress difference $N^{\text {neq }}$ on the wall boundary have been presented. In this section, a grid convergence study is conducted in order to identify the accuracy of the boundary condition. Also simulation results concerning the bulk flow are compared to the analytic solutions. The discretized lattice Boltzmann scheme of Eq. (2.1) after using the mapping $g_{i}=f_{i}+(1 / 2 \tau)\left(f_{i}-f_{i}^{e q}\right)$, and noting that $f_{i}^{e q}=g_{i}^{e q}$, is equal to:

$$
g_{i}\left(x+c_{i}, t+1\right)=g_{i}(x, t)+\frac{2}{2 \tau+1}\left[g_{i}^{e q}(x, t)-g_{i}(x, t)\right] .
$$

For the grid convergence study, the number of nodes in the $y$-direction varies and the specific values used are: [51, 101, 151, 301, 401, 801, 1050, 1500]. The Knudsen number 


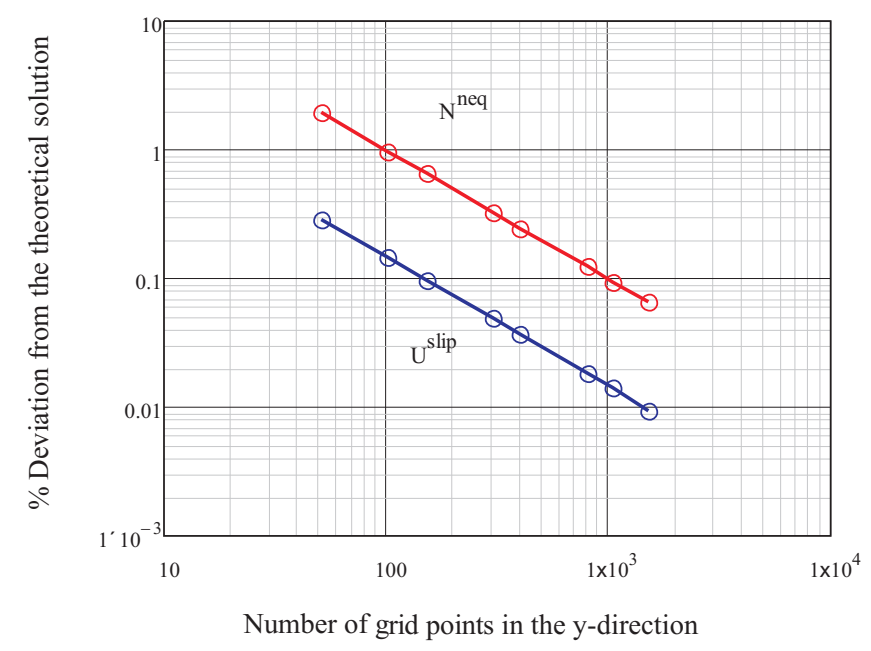

Figure 5: Grid convergence study for the micro-couette flow. The upper curve is the non-equilibrium normal stress difference $N^{n e q}$ while the lower curve is the slip velocity on the upper wall $U^{\text {slip }}$. Knudsen number is set to $K n=0.9$.

is set to $K n=0.9$. The results are illustrated in Fig. 5, where the slip velocity of Eq. (4.3) and the non-equilibrium normal stress difference of Eq. (4.4a) on the upper plate are considered. Both quantities follow the same trend and the order of convergence toward the analytic solution is one. This is in accordance with the slip velocity convergence study of [15]. The present simulation clearly shows that the obtained microflow behavior does not appear as discretization error. It is an inherent advantage of the lattice Boltzmann method. For the case where $K n=0.9$, the profiles along the $y$-direction for the velocity $U(y)$ in Fig. 6 and the non-equilibrium stress difference $N^{n e q}(y)$ in Fig. 7 are presented.

The non-equilibrium part of the diagonal term of the stress tensor $P_{x y}^{n e q}(y)$ is depicted in Fig. 8, and the non-equilibrium part of the heat flux along the $y$-direction $q_{y}^{\text {neq }}(y)$ in Fig. 9. In all the plots, the analytical solution is represented by a solid line, while the simulation results are the symbols. The lattice Boltzmann simulation results are found to be in excellent agreement with the exact solution for all studied quantities.

\section{Conclusions}

In this paper, the analytical solutions derived in [10] have been compared with the lattice Boltzmann simulation results. It is shown that a systematic study can provide a priori information about the quality and the validity of LB methods for micro-flows. It should be mentioned that even a small amount of nodes can deliver acceptable results for engineering applications. However, a grid convergence study reveals that the numerical implementation of the diffusive boundary condition as presented in the literature is first order accurate only $[8,9,16]$. A second order implementation of the diffusive boundary condition is needed for efficient modeling of the microflow. 


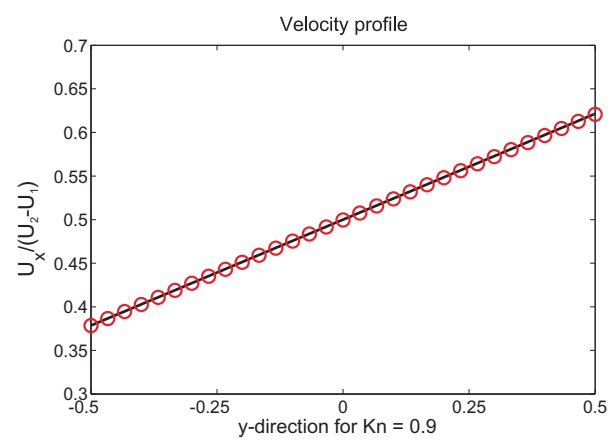

Figure 6: Comparison between analytic solution and LB simulations for the velocity profile at $K n=$ 0.9 . Line is the analytic solution; Symbols are LB simulation results.

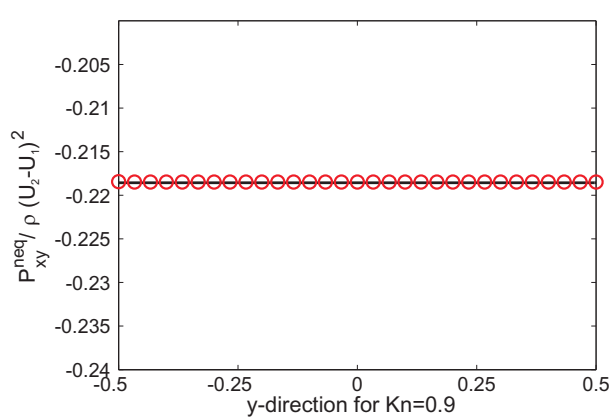

Figure 8: Comparison between analytic solution and LB simulations for the diagonal element of the Pressure tensor $P_{x y}$ at $K n=0.9$. Line is the analytic solution; Symbols are LB simulation results.

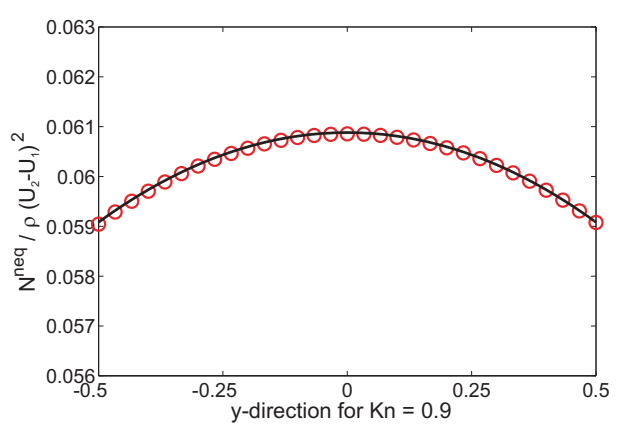

Figure 7: Comparison between analytic solution and LB simulations for the non-equilibrium stress difference $N^{n e q}$ profile at $K n=0.9$. Line is the analytic solution; Symbols are LB simulation results.

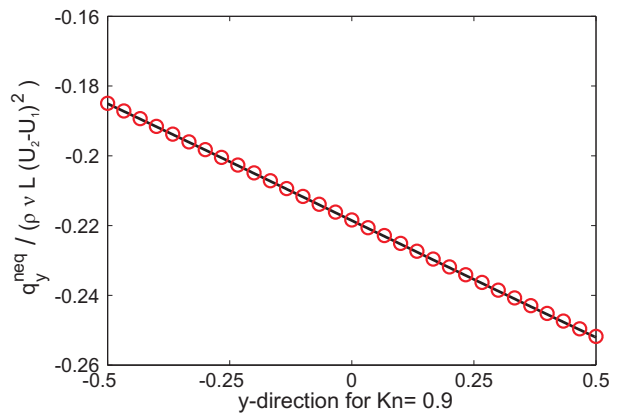

Figure 9: Comparison between analytic solution and LB simulations for the non-equilibrium heat flux $q_{y}^{\text {neq }}$ at $K n=0.9$. Line is the analytic solution; Symbols are LB simulation results.

\section{Acknowledgments}

The authors acknowledge useful discussions with Dr. I. V. Karlin. N. I. Prasianakis was supported by the BFE project 103078. S. Ansumali is thankful to Department of Science and Technology (DST), India for providing computational resources via Ramanujam Fellowship grant.

\section{References}

[1] A. Beskok, and G. E. Karniadakis, Microflows: Fundamentals and Simulation, Springer, Berlin, 2001.

[2] S. Succi, The Lattice Boltzmann Equation for Fluid Dynamics and Beyond, Oxford University Press, New York, 2001.

[3] S. Succi, Mesoscopic modeling of slip motion at fluid-solid interfaces with heterogeneous catalysis, Phys. Rev. Lett., 89 (2002), 064502. 
[4] B. M. Mognetti, and J. M. Yeomans, Capillary filling in microchannels patterned by posts, Phys. Rev. E., 80 (2009), 056309.

[5] M. Sbragaglia, and S. Succi, Analytical calculation of slip flow in lattice Boltzmann models with Kinetic boundary conditions, Phys. Fluids., 17 (2005), 093602.

[6] M. Sbragaglia, R. Benzi, L. Biferale, S. Succi, and F. Toschi, Surface roughnesshydrophobicity coupling in microchannel and nanochannel flows, Phys. Rev. Lett., 97 (2006), 204503.

[7] C. Kunert, and J. Harting, Roughness induced boundary slip in microchannel flows, Phys. Rev. Lett., 99 (2007), 176001.

[8] S. Ansumali, and I. V. Karlin, Kinetic boundary conditions in the lattice Boltzmann method, Phys. Rev. E., 66 (2002), 026311.

[9] S. Ansumali, I. V. Karlin, C. E. Frouzakis, and K. B. Boulouchos, Entropic lattice Boltzmann method for simulation of microflows, Phys. A., 359 (2006), 289-305.

[10] S. Ansumali, I. V. Karlin, S. Arcidiacono, A. Abbas, and N. I. Prasianakis, Hydrodynamics beyond Navier-Stokes: exact solution to the lattice Boltzmann hierarchy, Phys. Rev. Lett., 98 (2007), 124502.

[11] W. P. Yudistiawan, S. Ansumali, and I. V. Karlin, Hydrodynamics beyond Navier-Stokes: the slip flow model, Phys. Rev. E., 78 (2008), 016705.

[12] P. L. Bhatnagar, E. P. Gross, and M. Krook, A model for collision processes in gases. I. small amplitude processes in charged and neutral one-component systems, Phys. Rev., 94 (1954), 511-525.

[13] S. Ansumali, I. V. Karlin, and H. C. Ottinger, Minimal entropic Kinetic models for hydrodynamics, Europhys. Lett., 63 (2003), 798-804.

[14] Y. H. Qian, D. d'Humieres, and P. Lallemand, Lattice BGK models for Navier-Stokes equation, Europhys. Lett., 17 (1992), 479-484.

[15] S. H. Kim, H. Pitsch, and I. D. Boyd, Accuracy of higher-order lattice Boltzmann methods for microscale flows with finite Knudsen numbers, J. Comput. Phys., 227 (2008), 8655-8671.

[16] S. Ansumali, and I. V. Karlin, Consistent lattice Boltzmann methods, Phys. Rev. Lett., 95 (2005), 260605. 